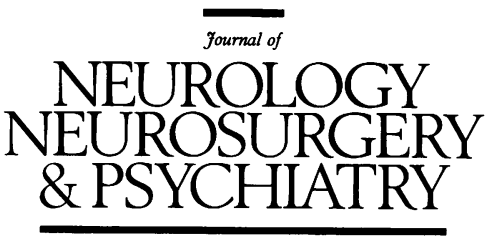

\title{
Editorial
}

\section{Alzheimer's disease: a treatment in sight?}

The concept of Alzheimer's disease as a treatable condition has a short history but already some would claim that we stand on the threshold of a new age in dementia care with the prospect of specific treatments for cognitive deficits in sight. Whether the efficacy of these treatments can live up to such high expectations still remains to be seen.

The economic and social consequences of Alzheimer's disease are considerable and largely unrecognised. Recent studies estimate the annual cost of dementia in the United Kingdom to be around $£ 1$ billion. ${ }^{1}$ Given the research investments made over the past decade by the pharmaceutical industry, the price of antidementia agents can be predicted to be high. Careful economic evaluation of the benefits of potential treatments will have to be made and the additional costs balanced against reduced expenditure on hospital and residential care. It is practically impossible, however, to estimate the magnitude of the greatest cost of dementia: the burden of care on families. Much of this burden results from non-cognitive symptomatology of dementia and treatments already exist for this. Unfortunately, rigorous clinical trials of commonly used psychotropic agents in dementia are limited both in number and in scope. ${ }^{2}$

The cognitive symptomatology of Alzheimer's disease has received most attention and investment of research with a wide variety of agents proposed as treatment. The complex class of compounds known as the nootropics and the related metabolically active compounds (including piratecam, vincarine, and idebenone) have had largely disappointing results in clinical trials (see for example, Gillis et $a l^{3}$ ). Although there has been a prolonged vogue for use of these classes of drugs in several countries (in particular in continental Europe and Japan); only specific neurotransmitter replacement treatments have managed to show beneficial effects in controlled trials in Alzheimer's disease.

Although Alzheimer's disease is likely to involve a variety of neurotransmitter defects, early evidence pointed towards a predominant loss of cholinergic function. ${ }^{45}$ This, together with the observed cognitive effects of experimental lesioning of the major cholinergic pathways of animals, led to a cholinergic hypothesis to explain the amnestic syndrome of Alzheimer's disease. ${ }^{6}$ It has to be said that demonstrations of further neurotransmitter deficiencies in Alzheimer's disease have done little to dampen the enthusiasm of those who support the hypothesis; perhaps because for the first time a rational approach to pharmacotherapy could be pursued. To increase cholinergic function in Alzheimer's disease various strategies have been employed, the most promising of which to date seems to have been the use of acetylcholinesterase inhibitors to decrease acetylcholine breakdown in the synaptic cleft. Enormous and largely unfounded excitement was engendered by the report from Summers $e t a l^{7}$ of a therapeutic benefit of one such anticholinesterase - tetrahydroaminoacridine (tacrine or THA). To note that this early trial was methodologically flawed is something of an understatement and the trial has been appropriately, and extensively, criticised. Despite this unfortunate beginning, some well designed and rigorous subsequent studies indicate that tacrine did in fact have a therapeutic benefit. ${ }^{8-10}$ Trials showing little or no benefit have also been published. ${ }^{112}$ Two recent large studies have overcome the logistic and design inadequacies of the early trials. ${ }^{1314}$ Together, these double blind placebo controlled studies included over 1000 patients with Alzheimer's disease. The earlier study titrated the dose of tacrine to a maximum of $80 \mathrm{mg}$ per day and the later one to $160 \mathrm{mg}$ per day. This study ${ }^{14}$ is the largest trial concluded to date on a putative compound for the treatment of Alzheimer's disease and can be taken as a sign of the scale of trials to come. Over 650 patients were included from 33 centres for a forced titration of tacrine in a double blind placebo controlled parallel group design. This is a prodigious effort, particularly when one considers that all patients had to meet the strict requirements of NINCDS criteria of Alzheimer's disease, to have only moderate cognitive impairment, and to be free from other relevant physical illness. Despite the prevalence of dementia, such patients are far from common. Both studies used the now obligatory global impressions of change in addition to the familiar mini mental state examination and ADAS measures together with assessments of activities of daily living. Such comprehensive outcome measures have been developed with the growth of clinical trials in dementia agents and illustrate the need for "gold standard" instruments to allow comparisons of data between clinical trials.

Neither study reported benefits from $80 \mathrm{mg}$ per day of tacrine on the mini mental state examination (MMSE) score although both showed efficacy on the ADAS cognitive scale at this dosage. Farlow et al ${ }^{13}$ found improvement on the global impression scale after 12 weeks of treatment at $80 \mathrm{mg}$ per day although this was not replicated in the later (larger) study. This later study ${ }^{14}$ did, 
however, show pronounced treatment effects using tacrine at the higher dosage of 120 or $160 \mathrm{mg}$ per day for a 30 week period. Improvements were seen in the global outcome measures, in the cognitive measures, and in the measurements of activities of daily living. Over the 30 weeks of the trial, patients on placebo declined by only 2.5 points on the ADAS scale measuring cognition. This is less than expected and may reflect the selection bias inherent in any trial requiring physically fit patients in the early stages of dementia. Despite this there was a mean difference between the placebo group and those receiving $160 \mathrm{mg}$ of tacrine of $2 \cdot 2$ points and $27 \%$ of the treated group improved by 4 or more points. Subjects in the placebo group declined by a mean of 0.9 points on the MMSE compared with no decline in the treated group overall and with $25 \%$ of the treated group improving by 3 points or more. Although relatively small in size, such a difference is likely to be welcomed by relatives and carers and should not be underrated. It is easy to argue that in the absence of other treatments, a modest benefit is better than no benefit at all.

Tacrine has side effects and can produce adverse reactions. Most troubling to the patient may be nausea, vomiting, or diarrhoea, occurring in up to a third of patients. More troubling to the treating physician is evidence of hepatotoxicity manifested as rises in serum transaminases. In the larger trial, half of the treated patients showed at least one alanine aminotransferase concentration above the normal upper limit and a third had concentrations more than three times the upper limit. Such disturbances of liver function are not permanent and return to normal on stopping treatment in most cases, allowing rechallenge to good effect in many instances.

Despite evidence of efficacy, tacrine has not been approved for use in the United Kingdom. As the decisions of the Committee of Safety of Medicine are unpublished, we cannot be certain of the reasons behind this, but presume that it results from concern regarding cost/benefit analyses. Cost/benefit analyses may, of course, relate to the clinical costs of adverse consequences and side effects as much as to any financial costs just as benefits can be measured in social and financial terms as much as in improvements in personal health. We are disappointed that because the deliberations of the licensing authorities are unpublished, the wider medical and scientific community is unable to evaluate which criteria have been instrumental in refusing a licence. Tacrine has, however, been approved by the rigorous procedures of the American Federal Drugs Administration (possibly influenced by active lobbying by groups representing patients in the United States), in some European countries, and elsewhere around the world. The effect of routine clinical use and postmarketing surveillance in these countries will prove instructive. Other anticholinesterases are in development and at the clinical trial stage. Each will be judged against tacrine in terms of placebo controlled efficacy and side effect profile. Perhaps the best that can be hoped for is a drug that is somewhat more efficacious and somewhat less hepatotoxic.

Use of drugs such as tacrine represents a palliative approach to the symptoms of Alzheimer's disease and as such the benefits will always be modest. Whereas such modest benefits are eagerly welcomed by carers and relatives in the absence of anything better, other strategies will need to be examined in the search for a preventative treatment or cure. Strategies such as trophic factor replacement therapies or attempts to intervene in the molecular pathogenesis are under development and increasingly the pharmaceutical industry is looking towards such approaches to develop a specific and rational treatment.

The era of designer drugs for Alzheimer's disease is not yet upon us, however, and is unlikely to be so for some decades. Perhaps this is to be welcomed; the prospect of any effective treatment for the cognitive impairment of Alzheimer's disease is likely to have a very considerable impact upon the delivery of old age psychiatry services and we, like our patients, need time to adjust. SIMON LOVESTONE

Section of Old Age Psychiatry, ROBERT HOWARD

Institute of Psychiatry,

De Crespigny Park, London SE5 8AF, UK

Correspondence to: Dr Lovestone.

1 Fenn P, Gray A. Alzheimer's disease; the burden of illness in England Health Trends 1993;25:31-7.

2 Burns A. Treatment of non-cognitive features of dementia. In: Levy $R$, Howard R, Burns A, eds. Treatment and care in old age psychiatry. Hampshire: Wrightson, 1993.

3 Gillis JC, Benfield P, McTavish D. Idebenone: a review of its pharmacodynamic and pharmakinetic properties and therapeutic use in agedynamic and pharmakinetic properties and therapeutic
related cognitive disorders. Drugs Aging 1994;5:133-52.

4 Whitehouse PJ, Price DL, Clarke AW, Coyle JT, Delong DE, Cantalello MG. Alzheimer's disease: evidence for selective loss of cholinergic neurones in the nucleus basalis. Ann Neurol 1981;10:122-6.

5 Davies P, Maloney AJF. Selective loss of central cholinergic neurones in Alzheimer's disease. Lancet 1976;2:1403.

6 Barthus RT, Dean RL, Beer B, Lipper AS. The cholinergic hypothesis of geriatric memory dysfunction. Science 1982;217:408-17.

7 Summers WK, Majovski LV, Marsh GM, Tachiki K, Kling A. Oral tetrahydroaminoacridine in long-term treatment of senile dementia, Alzheimer type. N Engl $₹$ Med 1986;315:1241-5.

8 Eagger SA, Levy R, Sahakian BJ. Tacrine in Alzheimer's disease. Lancet 1991;337:989-92.

9 Davis KL, Fowle LJ, Gamzu ER, Davis CS, Woolason RF, Gracon SI, et al. A double-blind placebo-controlled multicenter study of tacrine for al. A double-blind placebo-controlled multicenter s
Alzheimer's disease. $N$ Engl $\mathcal{F}$ Med 1992;327:1253-9.

10 Wilcock GK, Surmon DJ, Scott M, Boyle M, Mulligan K, Neubauer KA, et al. An evaluation of the efficacy and safety of tetrahydroaminoacridine (THA) without lecithin in the treatment of Alzheimer's disease. Age Ageing 1993;22:316-24.

11 Gauthier S, Bouchard R, Lamontagne A, Bailey P, Bergman H, Ratner J, et al. Tetrahydroaminoacridine-lecithin combination treatment in patients with intermediate-stage Alzheimer's disease. $N$ Engl $\mathcal{f} \mathrm{Med}$ 1990;322:1272-6.

12 Chatellier G, Lacomblez I. Tacrine (tetrahydroaminoacridine: THA) and lecithin in senile dementia of the Alzheimer type: a multicentre trial. $B M \mathcal{F}$ 1990;300:495-9.

13 Farlow M, Gracon SI, Hershey LA, Lewis KW, Sadowsky CH, DolanUreno J. A controlled tríal of tacrine in Alzheimer's disease. $\mathfrak{F A M A}$ 1992;268:2523-9.

14 Knapp MJ, Knopman DS, Solomon PR, Pendlebury WW, Davis CS, Gracon SI. A 30-week winderwise control trial of high dose tacrine in patients with Alzheimer's disease. $\mathcal{F} A M A$ 1994;271:985-91. 